\title{
Optimal Debulking
}

National Cancer Institute

\section{Source}

National Cancer Institute. Optimal Debulking. NCI Thesaurus. Code C128096.

Visible residual tumor less than or equal to one centimeter in diameter after cytoreductive surgery. 